\title{
Dermatomyositis and malignancy: should all patients with dermatomyositis undergo malignancy screening?
}

\author{
Urmi Khanna ${ }^{1}$, Fabrizio Galimberti ${ }^{2}$, Yumeng $\mathrm{Li}^{2}$, Anthony P. Fernandez ${ }^{1,3} \wedge$ \\ ${ }^{1}$ Department of Dermatology, Cleveland Clinic, Cleveland, Ohio, USA; ${ }^{2}$ Department of Dermatology and Cutaneous Surgery, University of Miami \\ Miller School of Medicine, Miami, Florida, USA; ${ }^{3}$ Department of Pathology, Cleveland Clinic, Cleveland, Ohio, USA \\ Contributions: (I) Conception and design: AP Fernandez; (II) Administrative support: U Khanna, AP Fernandez; (III) Provision of study materials or \\ patients: All authors; (IV) Collection and assembly of data: All authors; (V) Data analysis and interpretation: All authors; (VI) Manuscript writing: All \\ authors; (VII) Final approval of manuscript: All authors. \\ Correspondence to: Anthony P. Fernandez, MD, PhD. Director of Medical Dermatology, W.D. Steck Chair of Clinical Dermatology, Clinical Assistant \\ Professor, Cleveland Clinic Lerner College of Medicine; Departments of Dermatology and Pathology, Cleveland Clinic, 9500 Euclid Avenue, A61, \\ Cleveland, Ohio 44195, USA. Email: fernana6@ccf.org.
}

\begin{abstract}
Dermatomyositis is an autoimmune disease that occurs in association with underlying malignancy in a subset of patients. Given this association, diagnosis of dermatomyositis typically triggers malignancy screening. Although various malignancy screening protocols have been proposed, none have been extensively studied or taken into account prevalence of dermatomyositis-associated malignancies. We utilized peer-reviewed manuscripts identified by a Medline search from May 2000 to April 2020 to present a focused review concerning the association between dermatomyositis and malignancy, and controversies related to screening for malignancies most commonly occurring in dermatomyositis patients. This information was then synthesized to propose a rational strategy for approaching malignancy screening in dermatomyositis patients. Our review supports that risk of malignancy in dermatomyositis patients is wellestablished. However, the subset of dermatomyositis patients in whom the benefits of malignancy screening outweigh the risks of harm is unknown. Additionally, an evidence-based malignancy screening protocol for dermatomyositis patients that optimizes the risk:benefit ratio does not exist. Given the clear harms that can result, we propose that shared decision-making strategies be implemented to determine whether pursuit of malignancy screening conforms with dermatomyositis patients' desires and values. Physicians should be clear about potential risks and benefits of malignancy screening, and discuss clinical and serologic features present that may suggest/refute underlying malignancy during conversations aimed at shared decision-making. Research is greatly needed to determine which dermatomyositis patients warrant malignancy screening, which tests should be performed, and the intensity with which they should be ordered. Only after such work is done can malignancy screening in dermatomyositis patients be considered to have high value.
\end{abstract}

Keywords: Dermatomyositis; malignancy; screening; shared-decision; harms

Submitted Jul 12, 2020. Accepted for publication Nov 10, 2020.

doi: $10.21037 / \mathrm{atm}-20-5215$

View this article at: http://dx.doi.org/10.21037/atm-20-5215

\footnotetext{
^ ORCID: 0000-0003-4490-8427.
} 


\section{Introduction}

Dermatomyositis (DM) is an idiopathic inflammatory myopathy characterized mainly by myositis and skin findings. An association between underlying malignancy and DM in adults has been extensively studied and is well-known. Given this association, it is common for clinicians to consider screening patients for underlying malignancy when making a diagnosis of DM. However, it is unclear whether all adult patients with DM should undergo malignancy screening. Here we review evidence surrounding the association between malignancy and $\mathrm{DM}$, evidence concerning potential harms of malignancy screening tests, and propose a strategy concerning how to approach malignancy screening in DM patients based on this evidence.

\section{Methods}

A Medline search of the English literature from May 2000 to April 2020 was performed using the terms "malignancy", "meta-analysis", "predictors of malignancy", "malignancy imaging", "malignancy detection", and "malignancy screening" in combination with "dermatomyositis". Prospective studies, retrospective cohort studies, metaanalyses, and reviews with titles and abstracts suggesting presence of high-quality information were eligible for inclusion and their references reviewed by the authors to identify additional studies of interest. All eligible articles were then reviewed by the authors, who decided whether or not to include them in this review. Peer-reviewed literature concerning malignancy screening risks and harms were also reviewed, and research results pertinent to malignancy in the setting of DM are included here.

\section{Results}

\section{Association between malignancy and DM}

A link between malignancy and DM is well established. Numerous case series and population-based studies have confirmed this association, although estimation of malignancy incidence among DM patients varies from $15 \%$ to $27 \%(1,2)$. Current evidence supports that the risk and incidence of malignancy in the clinically amyopathic DM population is similar to that of the classic DM population (3-5). However, in a recent single academic center DM cohort, malignancy risk in clinically amyopathic DM patients was considerably lower than most other reported cohorts (1.7\% within 2 years of DM diagnosis; $5.4 \%$ within 5 years) (6). Importantly, risk of malignancy primarily relates to patients with adult-onset DM subtypes and not juvenile-onset DM subtypes (7).

The temporal association between adult-onset DM (hereafter referred to simply as DM) and malignancy has been reported in numerous cohorts. A recent study involving two large United States (US) cohorts totaling 400 DM patients found that $72 \%$ of all malignancy diagnoses occurred in the 5 years preceding or following DM symptom onset, with the highest frequency of malignancies occurring in the 12 months following DM symptom onset (3). An additional study reported that $64 \%$ of patients developed $\mathrm{DM}$ in the same year as the malignancy was discovered (8). Another US study found malignancy was most often detected within 1 year prior or after DM diagnosis (9), while yet another found malignancy was most often found at time of DM diagnosis or within 12 months following diagnosis (6).

Some studies have found that DM patients maintain an increased risk of malignancy compared to the general population for at least 5 years following DM diagnosis $(3,10,11)$. A decreasing trend of malignancy risk has been noted over this 5 -year time period, with the greatest standardized incidence ratio (SIR) during the first year after the diagnosis of DM. Buchbinder et al. reported an SIR of 4.4 during the first year after DM diagnosis, 3.4 between years 2 to 3, 2.2 between years 4 to 5 , and 1.6 beyond 5 years (10). A large meta-analysis from Canada revealed the SIR of malignancy among DM patients to be 17.29 [95\% confidence interval (CI), 11.08-26.99] in the first year, 2.70 (95\% CI, 1.96-3.72) from 2 to 5 years, and 1.37 (95\% CI, 1.27-1.48) after 5 years (11). Many authors believe a window of time one can reasonably consider a true association between DM and malignancy is approximately 3 years, and a significant percentage of published literature concerning DM and malignancy uses this time period as a cut-off for inclusion/exclusion criteria of cohort patients.

A wide variety of malignancies have been reported in association with $\mathrm{DM}$ in published medical literature. However, two meta-analyses have investigated the association of DM with tissue-specific malignancies in attempts to determine which malignancies actually occur with greater incidence in this population $(12,13)$. Yang et al.'s meta-analysis included 20 cohort studies from 1998 to 2013 with 882 malignancies in 7,374 DM patients and revealed increased risk for ovarian, breast, lung, colorectal, cervical, bladder, pancreatic, esophageal, nasopharyngeal and renal 
Table 1 Malignancies with increased incidence in dermatomyositis patients compared to the general public*

Malignancy type
Lung
Breast
Ovary
Colorectal
Cervical
Bladder
Nasopharyngeal
Esophageal
Kidney
Lymphatic
Hematopoietic

*, based on results of 2 meta-analyses $(11,12)$.

cancers for DM patients (13). Stratified analysis within this study showed higher malignancy risk in males compared to females (13). Olazagasti et al.'s meta-analysis included 10 cohort studies from 1992 to 2013 with 400 malignancies in 2,420 DM patients and revealed increased risk of lung, ovarian, breast, colorectal, lymphatic/hematopoietic, bladder, cervical, pancreatic and esophageal cancers among DM patients (12). When combining results of these metaanalyses, there appear to be 11 malignancy types that patients with DM generally demonstrate an increased risk for harboring/developing compared to the general population (Table 1).

\section{Clinical predictors of malignancy in DM}

Diverse clinical factors have been proposed to be predictive or protective of malignancy in DM patients (Table 2). A retrospective cohort of $121 \mathrm{DM}$ patients (29 with malignancy-associated DM) identified age $>52$ years at diagnosis, skin necrosis, periungual erythema, C4 hypocomplementemia, and rapid onset of skin/muscle symptoms as factors associated with malignancy, whereas lymphopenia $\left(<1,500 / \mathrm{mm}^{3}\right)$ was protective of malignancy (14). Other studies have reported interstitial lung disease (ILD) as protective against malignancy (15-17). Two metaanalyses $(18,19)$ reported increased malignancy risk among DM patients was associated with age $>45$ years, cutaneous necrosis, male sex, anti-TIF-1 $\gamma$ (anti-p155) antibody,
Table 2 Clinical and laboratory predictors of malignancy associated $\mathrm{DM}$

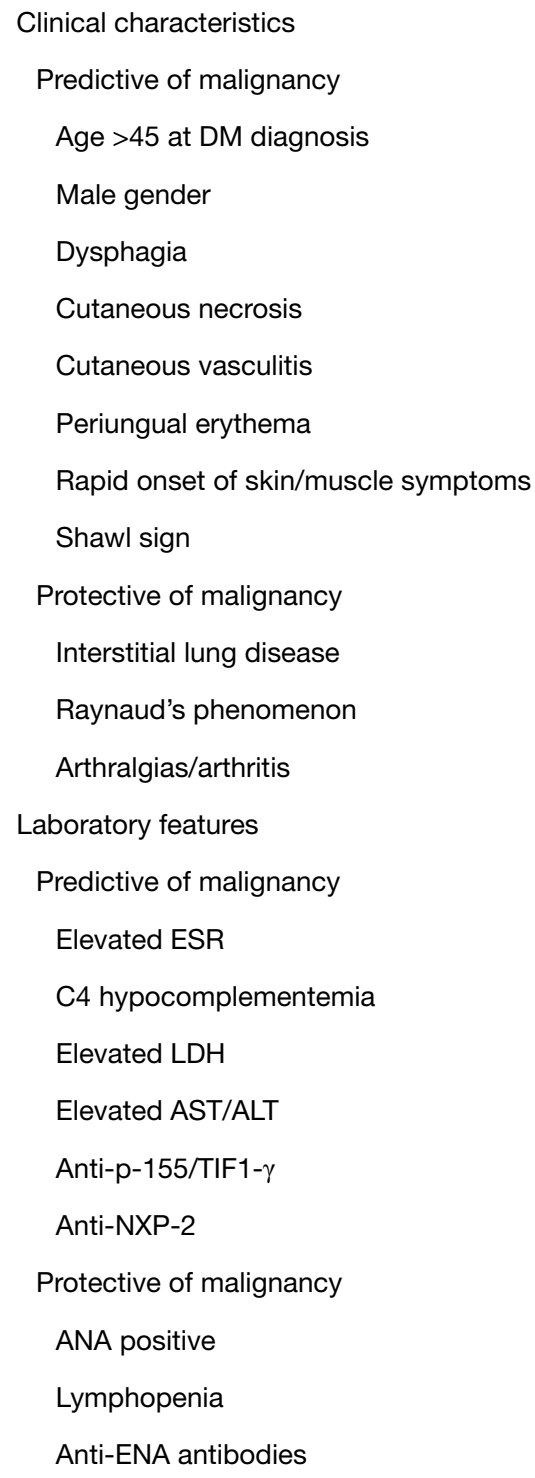

DM, dermatomyositis; ESR, erythrocyte sedimentation rate; $\mathrm{LDH}$, lactate dehydrogenase; TIF1- $\gamma$, transcription intermediary factor-1 gamma; NXP-2, nuclear matrix protein 2; ANA, antinuclear antibody; AST, aspartate aminotransaminase; ALT, alanine aminotransaminase; ENA, extractable nuclear antigen.

dysphagia, and elevated erythrocyte sedimentation rate (ESR) (>40 mm/hour), whereas presence of ILD, Raynaud's phenomenon, and anti-extractable nuclear antigen antibodies (anti-ENA) were associated with decreased malignancy risk $(18,19)$. As lymphopenia, anti-ENA antibodies, Raynaud's and ILD are common in autoimmune disease, lack of malignancy in their presence may signify a 
unique pathophysiology for idiopathic, "autoimmune" DM compared to malignancy-associated DM.

Myositis-specific autoantibodies (MSA) and myositisassociated autoantibodies (MAA) are found in 40-60\% of DM patients (20). Numerous studies have highlighted roles for MSA/MAA as malignancy predictors in DM. In particular, anti-TIF- $1 \gamma$ antibodies (anti-p155) were shown to be significantly associated with malignancy in a metaanalysis including six studies with a combined 312 patients [odds ratio $(\mathrm{OR})=27.26$ ] (21). A cross-sectional study of 103 DM patients also reported significant association between anti-TIF- $1 \gamma$ and malignancy $(\mathrm{OR}=8.0)(20)$. Notably, $0 / 16$ malignancy-associated DM patients in this study tested positive for an additional MAA/MSA antibody and the combination of a negative myositis panel and positive antiTIF- $1 \gamma$ had both $100 \%$ sensitivity and negative predictive value (NPV) for malignancy-associated DM (20). The results of a recent systematic review reported the OR of malignancy for patients with anti-TIF1- $\gamma$ positivity is 9.37 (95\% CI, 5.37-16.34) (22). Of note, the United Kingdom (UK) MyoNet study reported that malignancies in DM patients with anti-TIF1- $\gamma$ antibodies occurred exclusively within 3 years before/after DM onset, with no malignancies detected within the subsequent 7.5 years (23).

Studies have also demonstrated a correlation between anti-TIF1- $\gamma$ antibody-associated malignancy and age. Individuals with a DM diagnosis at age $>40$ years and positive TIF1- $\gamma$ antibodies showed an elevated risk $(75 \%)$ of cancer $(23,24)$. Additionally, the distribution of cancer sites may vary according to anti-TIF1- $\gamma$ antibody status. Female DM patients with anti-TIF1- $\gamma$ antibodies have been found to have an increased risk of ovarian cancer $(23,25,26)$.

Autoantibodies against nuclear matrix protein, NXP-2, were associated with malignancy $(\mathrm{OR}=2.5)$ in a study of 213 DM patients (27). However, stratification by sex revealed this to be true only for males $(\mathrm{OR}=5.78)(27)$. Presence of either anti-NXP-2 or anti-TIF1- $\gamma$ antibodies was highly associated with malignancy $(\mathrm{OR}=4.7)$ even after adjusting for age and sex, whereas absence of both was protective against malignancy (27).

Whether certain biopsy findings are associated with malignancy-associated DM has also been investigated. Uchino et al. (28) investigated muscle biopsies from 54 DM patients (43 with malignancy-associated DM). The majority (69\%) showed typical DM myositis histology (28). Although no malignancy association with endomysial infiltrationtype or perivascular infiltration-type was observed, the proportion of rare-infiltrative type was significantly higher in malignancy-associated DM compared to nonmalignancy-associated $\mathrm{DM}$ patients $(\mathrm{P}=0.0345)$ (28). To our knowledge, no histologic clues to malignancy-associated DM have been reported in skin biopsies.

\section{Radiologic screening modalities}

Few studies have investigated specific imaging modalities for DM malignancy screening. A role for screening with CT chest/abdomen/pelvis was shown in a retrospective analysis of $33 \mathrm{DM}$ patients, 13 of which were found to have malignancies (15). However, routine screening failed to identify four malignancies and screening not supported by physical exam (PE) and/or symptoms was positive in only $13 \%$ of cases (15). On the other hand, several recent cohort studies have found that CT scans were the most common test that detected an underlying malignancy when DM patients underwent malignancy screening $(3,9)$.

A single prospective study compared whole-body [18F] fluorodeoxyglucose positron emission tomography/ computed tomography (FDG-PET/CT) to "conventional" malignancy screening among DM patients (29). In this study, conventional screening included CT thorax/ abdomen, mammography, gynecological examination, pelvic ultrasound, and tumor marker analysis [CA125, CA19.9, carcinoembryonic antigen, prostate-specific antigen (PSA)] (29). Nine of 55 patients were diagnosed with malignancy. Notably, FDG-PET/CT and conventional screening had similar overall positive and NPVs for malignancy among DM patients (92.7\%) (29).

\section{The problem with malignancy screening in DM patients}

As the association between DM and underlying malignancy in a subset of patients seems clear, a diagnosis of DM typically triggers a malignancy screening work-up. However, screening strategies vary significantly from institution to institution, from department to department within institutions, and even from physician to physician within the same department. Additionally, although various malignancy screening protocols have been recommended by many groups, none are truly evidence-based and clearly take into account prevalence of DM-associated malignancies and/ or published screening guidelines for specific malignancies. Furthermore, a mortality benefit from screening DM patients for malignancy, the gold standard for proving screening value, has never been demonstrated. All of the above throw into question the risk:benefit ratio and overall 
value of malignancy screening in DM patients.

It is critically important for clinicians to recognize that malignancy screening tests are not entirely beneficial, but carry with them considerable potential for harm. However, empirical analyses show that primary screening studies pay little attention to the harms of performing screening. Of 57 screening studies examined in one report, only $7 \%$ quantified overdiagnosis and just $4 \%$ reported the rate of false positive results (30). Luckily, recognition and research evaluating risks and harms of screening tests, as well as the perception of screening tests from both patient and physician points of view, has been abundant and informative over the past decade.

Research has reproducibly demonstrated that one of the most significant causes of harm from malignancy screening tests is overdiagnosis, or the diagnosis of a (histologically confirmed) malignancy through screening that would not otherwise have been diagnosed or caused symptoms in a person's lifetime had screening not been done (31,32). Overdiagnosis is well-documented in screening strategies for numerous malignancies, including some of those found to occur with high incidence in the setting of DM, such as breast cancer (11-22\% overdiagnosis) $(33,34)$, lung cancer (15-25\%) (35), and renal cancer (42-58\%) $(32,36)$. In one study researchers found that as many as $33 \%$ of invasive breast cancer diagnoses by mammography represented overdiagnoses (37). A British analysis found that incidence of 10 of the 20 most common malignancies in the UK has increased by $>50 \%$ in both sexes since the 1980 s after screening protocols were implemented, including breast, kidney, cervical, and uterine carcinomas and non-Hodgkin's lymphomas, raising significant concern for widespread overdiagnosis (38). Although diagnosing any malignancy by screening may appear beneficial on the surface to poorly informed clinicians and patients, only harm can ensue when overdiagnosis occurs since the malignancy is not destined to ever hurt the patient. Harm may result from overtreatment (surgery, radiotherapy, endocrine therapy, and chemotherapy), significant psychologic impact to the patient and his/her family, and from significant negative financial impact to the patient, the patient's family and the healthcare system.

Other commonly recognized potential sources of harm from malignancy screening include false positive results and complications from testing. The culmination of various sources of harm can be significant enough to negate any benefits of malignancy screening. A systematic review of meta-analyses of cancer screening trials found that 3 of 10
(30\%) showed reductions in disease-specific mortality while failing to show reductions in overall mortality (39). One potential reason for such findings is that disease-specific mortality reductions could be offset by deaths caused by downstream effects of screening (39). Such "off-target deaths" are hypothesized to be caused by a variety of sources of harm, including complications of the screening test itself, complications of diagnostic tests following the screening test, over-detection and treatment of precancerous lesions, overdiagnosis of malignancy, false-positive results, and overtreatment $(31,40)$.

One of the most prominent examples underscoring the importance of recognizing potential harms of malignancy screening is the evolution of PSA testing in men. In 1992, the American Cancer Society (ACS) recommended annual PSA testing for men beginning at age 50 years (41). However, two large trials performed 20 years later showed PSA screening in asymptomatic men had little/no effect on mortality $(42,43)$. Soon after this, the European Randomized study of Screening for Prostate Cancer (ERSPC) trial revealed that 37 men will be diagnosed with prostate cancer for every death avoided (44), supporting that a significant number of men are overdiagnosed and overtreated with this screening strategy. Furthermore, $20 \%$ to $40 \%$ of men treated for prostate cancer will develop incontinence or erectile dysfunction (45). In light of evidence from these studies strongly supporting that risks and harms of PSA screening of asymptomatic men outweighed the potential benefits, the US Preventive Services Task Force (USPSTF) repeated its previous recommendation against screening and the ACS changed its stance and recommended that health care providers discuss risks and benefits of PSA screening with men $>50$ years of age so that they can make an informed decision $(45,46)$. Realization that harms may outweigh benefits has also resulted in reversal or abandonment of other malignancy screening campaigns in the past, including chest radiography screening for lung cancer and urine testing for neuroblastoma (30).

Despite abundant evidence demonstrating significant potential harm for most screening tests, research has shown that both patients and clinicians have a tendency to overestimate the benefits of screening tests, implying a poor recognition of the potential risks. In a systematic review, researchers found that patients more often overestimated benefits and underestimated harms of medical interventions, including screening tests (47). In a study examining patients' understanding of malignancy screening, only $19.7 \%$ of respondents understood that screening test harms may 
outweigh the benefits (48). On the clinician side, nearly all patients in one study reported that their clinicians addressed the benefits of cancer screening, but only approximately $25 \%$ reported that they received information about the risks (49). In a recent systematic review of clinician expectations of the benefits and harms of medical interventions, including screening tests, clinicians more often overestimated rather than underestimated benefits and underestimated rather than overestimated harms (50). Furthermore, research has shown many physicians would pursue equivocal incidental findings on tests contrary to or in the absence of evidence that they should be pursued (51).

Although questioning cost of screening tests is complex in the face of clear value in terms of saving lives, cost becomes important in the face of questionable benefits. For example, the cost of adopting CT screening for lung cancer by the Medicare population has been estimated to exceed $\$ 6$ billion per year, yet this screening strategy, which has demonstrated reduction in disease-specific mortality, has not demonstrated reduction in overall mortality. Failure to demonstrate reduction in overall mortality is possibly due to an overdiagnosis rate of $18 \%$ and major complications that are more than double the estimated number of lung cancer-specific deaths saved (30,52). Furthermore, low-dose CT scanning (LDCT) for lung cancer showed a $23.3 \%$ false-positive rate across three rounds of screening, and an $8.5 \%$ to $9.8 \%$ complication rate from invasive diagnostic procedures reported in the National Lung Screening Trial (NLST) for those who experienced false-positive screening results (53). Furthermore, the complication rates from diagnostic procedures following LDCT lung cancer screening in the real-world setting have been estimated to be significantly higher than those reported from clinical trials like the NLST, as are downstream costs related to complications (53). Costs related to harms of testing have also been explored and found to be significant for other malignancies, including breast cancer and colorectal carcinoma.

\section{Discussion}

\section{Should all DM patients be screened for malignancy?}

Clinicians making a diagnosis of DM in a patient may have a sincere and well-intentioned desire to immediately order a battery of malignancy screening tests and potentially "save" the patient's life. But should this simply be a knee-jerk reaction in clinicians who diagnose and treat DM patients?
The authors of this manuscript believe the answer is an emphatic, "no". Instead, we believe ordering malignancy screening tests in any DM patient requires the clinician to responsibly learn about both risks and benefits of these tests, and to engage the patient in decision-making.

With ongoing healthcare changes, it is increasingly recognized that malignancy screening guidelines should be of high-value, balancing benefit against both harms and cost. Recently, the High Value Care Task Force of the American College of Physicians (HVCTF-ACP) published a framework for cancer screening with highvalue care $(54,55)$. The motivation for their work was to promote high-value malignancy screening that optimizes balance between benefit, harm and cost in today's rapidly changing healthcare environment. High-value malignancy screening must take into account multiple factors, including identification of a population that warrants screening, which malignancies warrant screening, utilization of screening tests shown to be effective, and appropriate intensity of screening. Importantly, high-value malignancy screening must also recognize that screening tests can lead to harm and take into account potential harms, including adverse effects triggered/caused by testing, overdiagnosis and overtreatment. We believe recommendations for DMassociated malignancy screening should also follow these principles.

\section{What future work needs to be done to define "bigh-value" malignancy screening guidelines for DM patients?}

There is a tremendous amount of work to be done before malignancy screening can be considered "high-value" in the setting of DM. We believe one of the most important steps to this, as emphasized by the HVCTF-ACP in their framework, is to identify the subset of DM patients in whom malignancy screening has high value. To optimize the risk:benefit ratio of any screening test, it is well-known that it is important to define a high-risk subgroup. As only a minority will ever develop malignancy (15-27\%) identifying the subset of DM patients who warrant malignancy screening appears to be a feasible goal. Additional work to be done includes collecting data to, (I) minimize malignancy overdiagnosis in DM patients, (II) identify the prevalence of false positive malignancy diagnoses in DM patients, (III) assess patient morbidity/mortality associated with malignancy screening and (IV) calculate cost effectiveness of malignancy screening.

As summarized above, research has already laid the 
groundwork necessary for more focused projects that may eventually establish high-value malignancy screening guidelines within the setting of DM. For example, although malignancy risk may persist years after DM diagnosis, it has reproducibly been found to be highest within the first 12 months, supporting this is likely the time-period that will be associated with an optimal risk:benefit ratio and the highest screening value $(10,13)$. Furthermore, the clinical and laboratory factors that have been shown to predict or protect against underlying malignancy should be more meticulously explored to determine which, if any, can truly be used to further increase/decrease the level of clinical suspicion for underlying malignancies.

However, the important work ahead will also be fraught with significant challenges. For example, there is not likely to be a single "high-risk" malignancy definition that fits all DM patients. DM patients from Asian countries are much more likely to have nasopharyngeal cancer than DM patients from Europe and the US (56). Additionally, Japanese patients with DM may be particularly at risk for underlying gastric cancer (57). Thus, the malignancies that warrant screening for in different ethnic groups may not simply fall within the most common malignancies identified in above-mentioned meta-analyses, which included patients of varying ethnicity $(12,13)$. Furthermore, MAAs in different ethnic populations may infer different malignancy risks. In a study from Japan, the presence of anti-TIF$1 \gamma$ autoantibodies in DM patients were not only found to support a significantly higher risk of underlying malignancy, but also of more advanced malignancy, arguing against significant malignancy overdiagnosis (58). Thus, multiple factors, such as ethnicity, MAA status, gender, age, etc. may all need to be considered to define multiple "high-risk" DM patient subsets, and to identify specific malignancies that should be screened for in each subset.

Even more challenging may be the ability to identify malignancy overdiagnosis, false positive results, and other aspects of harm caused by malignancy screening in DM patients. None of these would be expected to simply be inferred from published studies detailing results from general patient populations, as DM defines a specific population. In theory, DM arising from an underlying malignancy occurs due to immunologic dysfunction related to a host immune system that is reacting to a malignancy because it recognizes potential harm to the host. This concept would be expected to have relevance concerning the extent of malignancy overdiagnosis, false positive results, and other potential harms of malignancy screening in DM patients.

One potential strategy to accrue the data needed to develop high-value malignancy screening guidelines in DM patients would be the establishment of an international prospective registry of DM patients that collects demographic, clinical, and outcome characteristics of DM patients who are screened for underlying malignancy. But, as seen with the evolution of PSA screening in men, identifying the pitfalls of malignancy screening and refining screening strategies will likely take many years. Additionally, once "high-value" malignancy screening guidelines have been developed, they must be tested prospectively for validation. Ultimately, ideal guidelines for malignancy screening in DM patients may not be possible. Nonetheless, well-designed objective research that attempts to improve the current landscape in which physicians individually decide who to screen and what tests to order in the absence of evidence driving these decisions would be expected to result in welcomed improvements in the care of DM patients.

\section{How should malignancy screening in DM patients currently be approached?}

Until this work is done and high-value malignancy screening guidelines are developed, should clinicians refrain from screening DM patients for malignancy? Again, we believe the answer is an emphatic, "no". The overall relative risk of malignancy is reported to be $\sim 5.5$ in DM patients and, notably, presence of malignancy is associated with poor prognosis in DM patients (59). Thus, malignancy screening is likely valuable and life-saving in some patients. But, instead of simply implementing malignancy screening protocols upon diagnosing a patient with DM, we believe clinicians should discuss the risks and benefits of malignancy screening with patients and make a shared decision concerning whether to pursue screening. This conversation could also include specifics about the patient's DM characteristics and take into consideration the risk factors summarized in Table 2.

A shared-decision approach is becoming more commonly recommended when considering screening for numerous malignancies, and takes into account the experience and expertise of patients, their priorities, and the particulars of their situation in helping them make an informed decision $(53,60-62)$. In addition to presenting the patient with facts about the benefits and risks of screening, clinicians facilitate an individualized decision-making process that aligns with 
the patient's preferences (40).

Ideally, clinicians caring for DM patients would be trained in patient-centered communication and shareddecision making. Such training could optimally prepare them to adjust their communication approach to individual patients, thus enabling decisions consistent with patients' needs, preferences, and values. This may be important, as different interpersonal and communication styles can affect decision-making outcomes (40). Additionally, decision aids to give to patients to help them understand risks and benefits may be developed and utilized. Although research concerning the use of patient decision aids has shown mixed results, a recent Cochrane review of 105 randomized trials of shared decision-making tools found that patient decision aids consistently improved patient knowledge of options and outcomes compared with control interventions and patient knowledge of risks $(31,63,64)$. Also, patients were clearer about what mattered most to them. Importantly, clinicians need to encourage discussion and collaborative decisionmaking with the patient to help arrive at a decision that is compatible with that individual's values and preferences, not simply present patients with current evidence concerning risks and benefits.

\section{Conclusions}

The link between DM and increased malignancy risk is well-established. However, whether or not some or all DM patients should be evaluated for underlying malignancy is unclear. Future research is needed to develop "highvalue" malignancy screening protocols that have favorable risk:benefit ratios in DM patient subsets. Such research should be done within the context of specific temporal periods when screening is thought to have the most value. Until then, we recommend incorporating shared decisionmaking methods in order to determine whether pursuing malignancy screening aligns with the patient's values and preferences. Importantly, physicians need to be honest about both potential benefits and harms of malignancy screening during such conversations, as well as about the unknowns concerning malignancy screening protocols and overall value in patients with DM.

\section{Acknowledgments}

Funding: This work was supported by the W.D. Steck Chair of Clinical Dermatology fund.

\section{Footnote}

Provenance and Peer Review: This article was commissioned by the Guest Editors (Drs. Richard D. Sontheimer, M. Kari Connolly, David F. Fiorentino, and Victoria P. Werth) for the series "Rheumatologic Skin Disease" published in Annals of Translational Medicine. The article has undergone external peer review.

Peer Review File: Available at http://dx.doi.org/10.21037/ atm-20-5215

Conflicts of Interest: All authors have completed the ICMJE uniform disclosure form (available at http://dx.doi. org/10.21037/atm-20-5215). The series "Rheumatologic Skin Disease" was commissioned by the editorial office without any funding or sponsorship. Dr. APF reports personal fees from AbbVie, grants and personal fees from Novartis, grants and personal fees from Mallinckrodt, other from Pfizer, other from Corbus, personal fees from Alexion, outside the submitted work. The other authors have no other conflicts of interest to declare.

Ethical Statement: The authors are accountable for all aspects of the work in ensuring that questions related to the accuracy or integrity of any part of the work are appropriately investigated and resolved.

Open Access Statement: This is an Open Access article distributed in accordance with the Creative Commons Attribution-NonCommercial-NoDerivs 4.0 International License (CC BY-NC-ND 4.0), which permits the noncommercial replication and distribution of the article with the strict proviso that no changes or edits are made and the original work is properly cited (including links to both the formal publication through the relevant DOI and the license). See: https://creativecommons.org/licenses/by-nc-nd/4.0/.

\section{References}

1. Sigurgeirsson B, Lindelöf B, Edhag O, et al. Risk of cancer in patients with dermatomyositis or polymyositis. A population-based study. N Engl J Med 1992;326:363-7.

2. Stockton D, Doherty VR, Brewster DH. Risk of cancer in patients with dermatomyositis or polymyositis, and followup implications: a Scottish population-based cohort study. Br J Cancer 2001;85:41-5.

3. Leatham H, Schadt C, Chisolm S, et al. Evidence 
supports blind screening for internal malignancy in dermatomyositis: data from 2 large US dermatology cohorts. Medicine (Baltimore) 2018;97:e9639.

4. Gerami P, Schope JM, McDonald L, et al. A systematic review of adult-onset clinically amyopathic dermatomyositis (dermatomyositis siné myositis): a missing link within the spectrum of the idiopathic inflammatory myopathies. J Am Acad Dermatol 2006;54:597-613.

5. Galimberti F, Li Y, Fernandez AP. Clinically amyopathic dermatomyositis: clinical features, response to medications and malignancy-associated risk factors in a specific tertiarycare-centre cohort. Br J Dermatol 2016;174:158-64.

6. Bowerman K, Pearson DR, Okawa J, et al. Malignancy in dermatomyositis: A retrospective study of 201 patients seen at the University of Pennsylvania. J Am Acad Dermatol 2020;83:117-22.

7. Morris P, Dare J. Juvenile dermatomyositis as a paraneoplastic phenomenon: An update. J Pediatr Hematol Oncol 2010;32:189-91.

8. Kundrick A, Kirby J, Ba D, et al. Positron emission tomography costs less to patients than conventional screening for malignancy in dermatomyositis. Semin Arthritis Rheum 2019;49:140-4.

9. Kooistra L, Ricotti C, Galimberti F, et al. Malignancyassociated dermatomyositis: retrospective case-control study from a single tertiary care center. J Am Acad Dermatol 2018;79:152-5.

10. Buchbinder R, Forbes A, Hall S, et al. Incidence of malignant disease in biopsy-proven inflammatory myopathy. A population-based cohort study. Ann Intern Med 2001;134:1087-95.

11. Qiang JK, Kim WB, Baibergenova A, et al. Risk of Malignancy in Dermatomyositis and Polymyositis. J Cutan Med Surg 2017;21:131-6.

12. Olazagasti JM, Baez PJ, Wetter DA, et al. Cancer risk in dermatomyositis: a meta-analysis of cohort studies. Am J Clin Dermatol 2015;16:89-98.

13. Yang Z, Lin F, Qin B, et al. Polymyositis/dermatomyositis and malignancy risk: a metaanalysis study. J Rheumatol 2015;42:282-91.

14. Fardet L, Dupuy A, Gain M, et al. Factors associated with underlying malignancy in a retrospective cohort of 121 patients with dermatomyositis. Medicine (Baltimore) 2009;88:91-7.

15. Sparsa A, Liozon E, Herrmann F, et al. Routine vs extensive malignancy search for adult dermatomyositis and polymyositis: a study of 40 patients. Arch Dermatol 2002;138:885-90.
16. Basset-Seguin N, Roujeau JC, Gherardi R, et al. Prognostic factors and predictive signs of malignancy in adult dermatomyositis. A study of 32 cases. Arch Dermatol 1990;126:633-7.

17. Ponyi A, Constantin T, Garami M, et al. Cancer-associated myositis: clinical features and prognostic signs. Ann NY Acad Sci 2005;1051:64-71.

18. Lu X, Yang H, Shu X, et al. Factors predicting malignancy in patients with polymyositis and dermatomyostis: a systematic review and meta- analysis. PLoS One 2014;9:e94128.

19. Wang J, Guo G, Chen G, et al. Meta-analysis of the association of dermatomyositis and polymyositis with cancer. Br J Dermatol 2013;169:838-47.

20. Chinoy H, Fertig N, Oddis CV, et al. The diagnostic utility of myositis autoantibody testing for predicting the risk of cancer-associated myositis. Ann Rheum Dis 2007;66:1345-9.

21. Trallero-Araguás E, Rodrigo-Pendás JÁ, SelvaO'Callaghan A, et al. Usefulness of anti-p155 autoantibody for diagnosing cancer-associated dermatomyositis: a systematic review and meta-analysis. Arthritis Rheum 2012;64:523-32.

22. Best M, Molinari N, Chasset F, et al. Use of antitranscriptional intermediary factor-1 gamma autoantibody in identifying adult dermatomyositis patients with cancer: a systematic review and meta-analysis. Acta Derm Venereol 2019;99:256-62.

23. Oldroyd A, Sergeant JC, New P, et al. The temporal relationship between cancer and adult onset antitranscriptional intermediary factor 1 antibody-positive dermatomyositis. Rheumatology (Oxford) 2019;58:650-5.

24. Fujimoto M, Hamaguchi Y, Kaji K, et al. Myositisspecific anti-155/140 autoantibodies target transcription intermediary factor 1 family proteins. Arthritis Rheum 2012;64:513-22.

25. Hill CL, Zhang Y, Sigurgeirsson B, et al. Frequency of specific cancer types in dermatomyositis and polymyositis: A population-based study. Lancet 2001;357:96-100.

26. Kang EH, Lee SJ, Ascherman DP, et al. Temporal relationship between cancer and myositis identifies two distinctive subgroups of cancers: impact on cancer risk and survival in patients with myositis. Rheumatology (Oxford) 2016;55:1631-41.

27. Fiorentino DF, Chung LS, Christopher-Stine L, et al. Most patients with cancer-associated dermatomyositis have antibodies to nuclear matrix protein NXP-2 or transcription intermediary factor $1 \gamma$. Arthritis Rheum 
2013;65:2954-62.

28. Uchino M, Yamashita S, Uchino K, et al. Muscle biopsy findings predictive of malignancy in rare infiltrative dermatomyositis. Clin Neurol Neurosurg 2013;115:603-6.

29. Selva-O'Callaghan A, Grau JM, Gámez-Cenzano C, et al. Conventional cancer screening versus PET/CT in dermatomyositis/polymyositis. Am J Med 2010;123:558-62.

30. Prasad V, Lenzer J, Newman DH. Why cancer screening has never been shown to "save lives" - and what we can do about it. BMJ 2016;352:h6080.

31. Davies L, Petitti DB, Martin L, et al. Defining, estimating, and communicating overdiagnosis in cancer screening. Ann Intern Med 2018;169:36-43.

32. Glasziou PP, Jones MA, Pathirana T, et al. Estimating the magnitude of cancer overdiagnosis in Australia. Med J Aust 2020;212:163-8.

33. Marmot MG, Altman DG, Cameron DA, et al. The benefits and harms of breast cancer screening: an independent review. Br J Cancer 2013;108:2205-40.

34. Nelson HD, Pappas M, Cantor A, et al. Harms of breast cancer screening: systematic review to update the 2009 U.S. Preventive Services Task Force Recommendation. Ann Intern Med 2016;164:256-67.

35. Patz EFJ, Pinsky P, Gatsonis C, et al. Overdiagnosis in low-dose computed tomography screening for lung cancer. JAMA Intern Med 2014;174:269-74.

36. Srivastava S, Koay EJ, Borowsky AD, et al. Cancer overdiagnosis: a biological challenge and clinical dilemma. Nat Rev Cancer 2019;19:349-58.

37. Jørgensen KJ, Gøtzsche PC. Overdiagnosis in publicly organised mammography screening programmes: systematic review of incidence trends. BMJ 2009;339:b2587.

38. Oke JL, O'Sullivan JW, Perera R, et al. The mapping of cancer incidence and mortality trends in the UK from 1980-2013 reveals a potential for overdiagnosis. Sci Rep 2018;8:14663.

39. Saquib N, Saquib J, Ioannidis JPA. Does screening for disease save lives in asymptomatic adults? Systematic review of meta-analyses and randomized trials. Int J Epidemiol 2015;44:264-77.

40. Breslau ES, Gorin SS, Edwards HM, et al. An individualized approach to cancer screening decisions in older adults: a multilevel framework. J Gen Intern Med 2016;31:539-47.

41. Mettlin C, Jones G, Averette H, et al. Defining and updating the American Cancer Society guidelines for the cancer-related checkup: prostate and endometrial cancers.
CA Cancer J Clin 1993;43:42-6.

42. Andriole GL, Crawford ED, Grubb RL 3rd, et al. Prostate cancer screening in the randomized prostate, lung, colorectal, and ovarian cancer screening trial: mortality results after 13 years of follow-up. J Natl Cancer Inst 2012;104:125-32.

43. Schröder FH, Hugosson J, Roobol MJ, et al. Prostatecancer mortality at 11 years of follow-up. N Engl J Med 2012;366:981-90.

44. Hayes JH, Barry MJ. Screening for prostate cancer with the prostate-specific antigen test: a review of current evidence. JAMA 2014;311:1143-9.

45. Moyer VA. Screening for prostate cancer: U.S. Preventive Services Task Force recommendation statement. Ann Intern Med 2012;157:120-34.

46. Smith RA, Manassaram-Baptiste D, Brooks D, et al. Cancer screening in the United States, 2014: a review of current American Cancer Society guidelines and current issues in cancer screening. CA Cancer J Clin 2014;64:30-51.

47. Hoffmann TC, Del Mar C. Patients' expectations of the benefits and harms of treatments, screening, and tests: a systematic review. JAMA Intern Med 2015;175:274-86.

48. Roberts MC, Ferrer RA, Rendle KA, et al. Lay beliefs about the accuracy and value of cancer screening. Am J Prev Med 2018;54:699-703.

49. Harris RP, Sheridan SL, Lewis CL, et al. The harms of screening: a proposed taxonomy and application to lung cancer screening. JAMA Intern Med 2014;174:281-5.

50. Hoffmann TC, Del Mar C. Clinicians' expectations of the benefits and harms of treatments, screening, and tests: a systematic review. JAMA Intern Med 2017;177:407-19.

51. Ganguli I, Simpkin AL, Colla CH, et al. Why do physicians pursue cascades of care after incidental findings? A national survey. J Gen Intern Med 2020;35:1352-4.

52. Roth JA, Sullivan SD, Goulart BHL, et al. Projected clinical, resource use, and fiscal impacts of implementing low-dose computed tomography lung cancer screening in Medicare. J Oncol Pract 2015;11:267-72.

53. Huo J, Xu Y, Sheu T, et al. Complication Rates and Downstream Medical Costs Associated With Invasive Diagnostic Procedures for Lung Abnormalities in the Community Setting. JAMA Intern Med 2019;179:324-32.

54. Harris RP, Wilt TJ, Qaseem Aet al. A value framework for cancer screening: advice for high-value care from the American College of Physicians. Ann Intern Med 2015;162:712-7.

55. Wilt TJ, Harris RP, Qaseem A, et al. Screening for cancer: Advice for high-value care from the American College of 
Physicians. Ann Intern Med 2015;162:718-25.

56. Ungprasert P, Leeaphorn N, Hosiriluck N, et al. Clinical features of inflammatory myopathies and their association with malignancy: a systematic review in Asian population. ISRN Rheumatol 2013;2013:509354.

57. Azuma K, Yamada H, Ohkubo M, et al. Incidence and predictive factors for malignancies in 136 Japanese patients with dermatomyositis, polymyositis and clinically amyopathic dermatomyositis. Mod Rheumatol 2011;21:178-83.

58. Ogawa-Momohara M, Muro Y, Mitsuma T, et al. Strong correlation between cancer progression and anti-transcription intermediary factor $1 \gamma$ antibodies in dermatomyositis patients. Clin Exp Rheumatol 2018;36:990-5.

59. András C, Ponyi A, Constantin T, et al. Dermatomyositis

Cite this article as: Khanna U, Galimberti F, Li Y, Fernandez AP. Dermatomyositis and malignancy: should all patients with dermatomyositis undergo malignancy screening? Ann Transl Med 2021;9(5):432. doi: 10.21037/atm-20-5215 and polymyositis associated with malignancy: a 21-year retrospective study. J Rheumatol 2008;35:438-44.

60. Rabi DM, Kunneman M, Montori VM. When guidelines recommend shared decision-making. JAMA 2020;323:1345-6.

61. Keating NL, Pace LE. Breast cancer screening in 2018: time for shared decision making. JAMA 2018;319:1814-5.

62. Caverly TJ, Meza R. Using risk models to make lung cancer screening decisions: evidence-based and getting better. Ann Intern Med 2019;171:669-70.

63. Montori VM, Kunneman M, Brito JP. Shared decision making and improving health care: the answer is not in. JAMA 2017;318:617-618.

64. Stacey D, Légaré F, Col NF, et al. Decision aids for people facing health treatment or screening decisions. Cochrane Database Syst Rev 2014;1:CD001431. 